\title{
Fatores determinantes para o desenvolvimento da Ortorexia Nervosa: uma revisão
}

\section{de literatura}

\author{
Determining factors for the development of Nervous Orthorexia: a literature review \\ Factores determinantes para el desarrollo de ortorexia nerviosa: revisión de la literatura
}

Recebido: 13/12/2021 | Revisado: 19/12/2021 | Aceito: 21/12/2021 | Publicado: 03/01/2022

\author{
Marta Ester da Silva \\ ORCID: https://orcid.org/0000-0001-5030-1520 \\ Faculdade de Comunicação, Tecnologia e Turismo de Olinda, Brasil \\ E-mail: marta.silva.ester@gmail.com \\ Gleyce Kelly de Araujo \\ ORCID: https://orcid.org/0000-0002-9388-7389 \\ Universidade Federal de Pernambuco, Brasil \\ E-mail: gleycearaujo.nutri@gmail.com \\ Maria Suzane da Silva Barbosa \\ ORCID: https://orcid.org/0000-0003-1995-8721 \\ Universidade Federal de Pernambuco, Brasil \\ E-mail: m.suzane.barbosa@gmail.com \\ Fabio Torres Cunha \\ ORCID: http://orcid.org/0000-0003-0238-7600 \\ Faculdade de Comunicação, Tecnologia e Turismo de Olinda, Brasil \\ E-mail: fabiologo1980@gmail.com \\ Danielle Cássia de Oliveira \\ ORCID: https://orcid.org/0000-0003-4154-2597 \\ Faculdade de Comunicação, Tecnologia e Turismo de Olinda, Brasil \\ E-mail: daniellecassiao@yahoo.com.br
}

\begin{abstract}
Resumo
Objetivo: Identificar as características individuais que podem levar o adulto a desenvolver ortorexia nervosa. Metodologia: Trata-se de uma pesquisa de revisão bibliográfica integrativa, onde foi efetuado buscas de publicações incluídos artigos nos anos de 2006 a 2020, dos quais estavam disponíveis na íntegra em acesso livre, onde abordavam questões sobre o tema proposto. Resultados: Estima-se que dentre a população geral, de alto risco estão (profissionais e área da saúde) entre 35\% e 57,8\% Alguns dos estudos também mostram um baixo nível de escolaridade. Além disso, pessoas que já sofreram algum tipo de transtorno/distúrbio alimentar indicam expor a ortorexia com mais ênfase. Conclusão: A maior prevalência em ortoréxicos são de profissionais da área da saúde, pessoas veganas, pessoas ligadas a causas de proteção animal e praticantes de exercícios físicos, tendo uma pequena porcentagem as pessoas fora desse ciclo. Entretanto, alguns dos estudos demonstram que existe um baixo nível de escolaridade entre os indivíduos com ortorexia nervosa, vale salientar que são necessários mais estudos para evidenciar tais fatos.
\end{abstract}

Palavras-chave: Comportamento alimentar; Dieta; Transtorno alimentar; Hábitos alimentares.

\begin{abstract}
Objective: To identify the individual characteristics that can lead the adult individual to develop orthorexia nervosa. Methodology: This is a review study integrative bibliography, where searches for publications including articles were carried out in the 2006 to 2020, of which they were available in full in free access, where they addressed questions on the proposed topic. Results: It is estimated at $6.9 \%$, and in high-risk groups are (professionals and health area) between 35\% and 57.8\%. Some of the studies also show a low level of education. Ademias, People who have already suffered some type of eating disorder / disorder indicate exposing orthorexia with more emphasis. Conclusion: A highest prevalence in orthorexics are health professionals, vegan people, people linked to animal protection causes and practitioners of physical exercises, having a small percentage of people outside this cycle. However, some of the studies demonstrate that there is a low level of education among individuals with orthorexia nervosa, it is worth to emphasize that more studies are needed to evidence such facts.
\end{abstract}

Keywords: Eating behavior; Diet; Eating disorder; Habits food.

\section{Resumen}

Objetivo: Identificar las características individuales que pueden llevar al individuo adulto desarrollar ortorexia nerviosa. Metodología: este es un estudio de revisión bibliografía integradora, donde se realizaron búsquedas de 
publicaciones incluyendo artículos en el 2006 a 2020, de los cuales estuvieron disponibles en su totalidad en acceso gratuito, donde abordaron preguntas sobre el tema propuesto. Resultados: Se estima en 6,9\%, y en grupos de alto riesgo son (profesionales y área de salud) entre el 35\% y el 57,8\%. Algunos de los estudios también muestran un bajo nivel de educación. Además, personas que ya han sufrido algún tipo de el trastorno / trastorno alimentario indica exponer la ortorexia con más énfasis. Conclusión: A mayor prevalencia en ortorexicos son profesionales de la salud, personas veganas, personas vinculadas a causas protectoras de animales y practicantes de ejercicio físico, teniendo un pequeño porcentaje de personas fuera de este ciclo. Sin embargo, algunos de los estudios demuestran que hay un bajo nivel de educación entre las personas con ortorexia nerviosa, es importante enfatizar que se necesitan más estudios para evidenciar tales hechos.

Palabras clave: Conducta alimentaria; Dieta; Desorden alimenticio; Hábitos alimentares.

\section{Introdução}

A transição nutricional trouxe consigo mudanças no padrão dietético, e consequentemente, condições que podem promover mudanças na composição corporal e na estética, desencadeando transtornos alimentares (Dell'osso et.al, 2016).

Com isso Dell'osso et.al, (2016), fala que os transtornos alimentares (TA) são definidos por uma inquietação permanente na alimentação ou no comportamento ligado à alimentação que resulta na ingestão ou na captação alterada de alimentos e que compromete consideravelmente a saúde física ou o funcionamento psicossocial. No meio se destacam os seguintes: Pica, transtorno de Ruminação, Ortorexia Nervosa (ON), Anorexia Nervosa (AN), Bulimia Nervosa (BN) e Transtorno de Compulsão Alimentar.

Logo, destaca-se a ortorexia nervosa $(\mathrm{ON})$ que a princípio foi relatada pelo médico americano Steven Bratman, em 1997, como uma fixação exacerbada pela saúde alimentar definida pela obsessão doentia com o alimento biologicamente puro, ocorrendo diversas restrições alimentares importantes. Os sujeitos com ON apresentam preocupação excessiva com a qualidade dos alimentos, sendo essa sua essencial preocupação, excluindo ao máximo o consumo dos alimentos que obtenham substâncias como corantes, conservantes, pesticidas, ingredientes transgênicos, gorduras menos saudáveis e excesso de sal e açúcar (Penaforte et al, 2019).

Visto que, os indivíduos com ON buscam abolir de sua alimentação, alimentos que não sejam orgânicos e se preocupam em abundância com o seu modo de preparo e origem da matéria-prima. Entretanto, a capacidade de alimentos ingerida e a perda de peso não são consideradas prioridades para os indivíduos com ON, muito embora a perda de peso seja apontada como um possível fator desencadeador desse desvio do comportamento alimentar (Penaforte et al, 2019 \& Aranceta, 2020).

Em vista disso, o tratamento adequado da ON permite impedir a sua complexidade e melhora a qualidade de vida do indivíduo com ON, diminuindo o estresse e a ansiedade. Existem ferramentas para diagnosticar ON, o questionário “ORTO 15" é uma delas, tendo como diagnóstico a presença de ON com um valor igual ou inferior a 40 pontos. Dado estes, que os pacientes geralmente não têm conhecimento de sua obsessão por alimentos. Logo, a colaboração de uma equipe profissional com médicos, psicólogos e outros profissionais é essencial para o tratamento, visando uma melhor qualidade de vida para o paciente (Cavero et al, 2015; Andreas et al, 2018 \& Bartrina,2008).

Devido ao grande aumento de casos com Ortorexia Nervosa em indivíduos que se intensificou nos últimos anos, é esperado atrair atenção para o tema e contribuir para a descoberta de novas hipóteses nos aparecimentos de ON. Dentro deste aspecto, esta pesquisa objetivou identificar as características individuais que podem levar o indivíduo a desenvolver Ortorexia nervosa.

\section{Metodologia}

Trata-se de uma pesquisa de revisão bibliográfica integrativa, onde foi efetuado buscas de publicações nas palavras chave: “comportamento alimentar”, “dieta”, “transtorno alimentar”, "hábitos alimentares”. Referente às características dos 
indivíduos ortoréxicos, nas plataformas de dados SciELO, PubMed, Capes e Lilex nos idiomas português, inglês e espanhol. Incluídos artigos nos anos de 2006 a 2020, dos quais estavam disponíveis na íntegra em acesso livre, onde abordavam questões sobre o tema proposto e excluindo artigos sem acesso livre, fora da temática proposta, fora da faixa de anos estipulados como também, artigos de resumos e resenhas.

\section{Resultados}

A presente pesquisa analisou um total de 109 artigos dentro das plataformas Pubmed, Scielo, Capes e Lilacs, na qual se obteve uma maior quantidade de artigos encontrados no Capes, e uma menor quantidade no Lilex. Todos os artigos estavam disponíveis com acesso livre, nos anos de 2006 a 2020. Na qual foram divididos em tópicos de acordo com o tema da pesquisa, como mostra as Tabelas 1 e 2.

Tabela 1: Quantidade de artigos encontrados por Plataforma.

\begin{tabular}{cccc}
\hline & BASE DE DADOS & TOTAL & INCLUSO \\
\hline PubMed & 24 & 6 & 18 \\
SciELO & 19 & 13 & 6 \\
Capes & 57 & 17 & 40 \\
Lilacs & 9 & 6 & 3 \\
TOTAL & 109 & 23 & 86 \\
\hline
\end{tabular}

Fonte: Autores.

Tabela 2: Síntese fundamental dos artigos abordados.

\begin{tabular}{llll}
\hline AUTOR/ANO & OBJETIVO & RESULTADOS & CONCLUSÃO
\end{tabular}

\section{ALÉN,2006}

ARANCETA 2007

GONÇALVES, 2008

POTYRALA, 2018
Demonstrar que os estudos de antropologia e oferecer novas maneiras de entender e interpretar a enfermaria, em de um caso de ortorexia nervosa.

Demonstrar conceitos científicos que demonstrem a obsessão pela comida saudável ao extremo se tornando uma patologia.

Avaliar a percepção corporal e a prevalência de sintomas de anorexia nervosa em estudantes universitários

Apresentar e comparar o conhecimento existente sobre o assunto

ortorexia nervosa e distúrbio alimentar noturno.
Pacientes com ortorexia,

de quem ditam as crenças e escolhas alimentares, eles rejeitam precisamente a ingestão de proteínas animais, que foram fundamentais para a evolução do homem.

Entre 318 médicos residentes em um hospital em Ancara, Turquia, estimou que $45,5 \%$ dos participantes obtiveram pontuação compatível com ortorexia no teste de triagem ORTO-15

$\mathrm{O}$ teste de imagem corporal evidenciou proporções elevadas de insatisfação com a forma corporal, em ambos os cursos: $75,8 \%$ e $78,2 \%$ para os cursos de nutrição e educação física, respectivamente.

A ortorexia ocorre em $0,5-1 \%$ da população, entre ambos os sexos com a mesma frequência, particularmente em artistas, desportistas, médicos e estudantes de medicina.
Muitos pacientes, com anorexia, bulimia ou ortorexia, transformaram sua vida em religião, estilo de vida, segundo eles, uma opção errada, mas uma escolha de acordo com o estudo.

Trabalhar com o ambiente imediato dos pacientes e promover a educação nutricional precoce são complementos essencias para se chegar à solução definitiva do problema.

Resultados evidenciaram proporções elevadas de insatisfação com a imagem corporal, em ambos os cursos.

Ortorexia desenvolve-se lenta e insidiosamente. Já a síndrome da alimentação noturna pode ser uma das causas de sobrepeso e obesidade, e no futuro, doenças metabólicas. O nível de estresse dos pacientes, que é um fator importante. 
MARTÍN et.al, Analisar o comportamento 2014

NEGRI et.al, 2011. Objetivou-se identificar em calouros as influências na escolha por nutrição e suas expectativas, através de estudo exploratório.

BARTHELS;

MEYER;

PIETROWSKY, 2018

VBARRADA; RONCERO, 2018

OLEJNICZAK et.al, 2017

DITTFELD et.al, 2017

GRAMAGLIA et.al, 2017

SOUZA, 2014

SILVEIRA et.al,2015 alimentares em uma amostra praticando exercícios físicos atividade.

Analisar o comportamento alimentar ortoréxico e contido em uma amostra de veganos e vegetarianos e uma amostra de indivíduos em dieta para perder peso

O objetivo deste estudo foi duplo. Primeiro, desenvolver e validar um novo questionário de ortorexia - a Escala Teruel de Ortorexia - e, segundo, analisar a associação com outras estruturas e distúrbios psicológicos

O objetivo de nossa pesquisa foi analisar comportamentos nutricionais

para a avaliação do risco de ortorexia

Descrever a relação entre vegetarianismo e ortorexia nervosa $(\mathrm{ON})$.

Avaliar a possível sobreposição entre ortorexia, medida com o Teste ORTO-15 e AN. Em italiano é poloneses.

Identificar comportamento de risco para o desenvolvimento de ortorexia nervosa em estudantes de Nutrição.

Este artigo objetiva expor, evidências que relacionem a
Comportamentos ortoréxicos e obsessivos foram encontrados em ambos os sexos, para alguns marcadores estudados.

Os dados analisados pela estatística descritiva indicaram o interesse em "educar pessoas à alimentação saudável" (26\%) e "ser profissão da área da saúde" (19\%) Ortorexia Nervosa ao Transtorno Obsessivo Compulsivo

Veganos e vegetarianos não diferem no comportamento alimentar ortoréxico, mas ambos os grupos pontuam mais no comportamento alimentar ortoréxico do que os indivíduos que consomem carne vermelha.

148 ofereceram respostas em um reteste 18 meses depois. Após iniciar com um banco inicial de 31 itens, foi proposto um teste bidimensional de ortorexia.

Observou-se que o número de refeições por dia

foi correlacionado com o tempo declarado gasto no planejamento de uma dieta

Com base nos resultados obtidos, o fanatismo por alimentos saudáveis é mais específico para vegetarianos do que para não vegetarianos.

Ambos os grupos italianos pontuaram significativamente superior aos poloneses no ORTO-15.

Participaram do estudo 150 alunas, com idade média de 23,21 anos. Observouse que a maioria das alunas estava eutrófica $(74 \%, \mathrm{n}=111)$, segundo a classificação do IMC

Não foram encontrados estudos que demonstrem a relação direta entre Ortorexia Nervosa e o Transtorno Obsessivo Compulsivo.
Os hábitos analisados mostram tendência à adoração corporal, mais próxima de uma condição patológica do que para um aspecto saudável, causando alterações na percepção, que resultam em dieta e alterações como comer distúrbios.

Contudo, foi importante mostrar aos calouros de nutrição as possibilidades de atuação profissional, seu papel na promoção da saúde e as diversidades de atuação, em sintonia com os avanços da ciência. Este estudo apresenta um novo
instrumento promissor que oferece possibilidades no estudo da $\mathrm{ON}$

Validações cruzadas adicionais do instrumento

deve ser feito para testar a estabilidade dos resultados em comunidades e amostras clínicas

Devido informações insuficientes sobre ortorexia, é essencial realizar pesquisas adicionais para determinar as características dos grupos de alto risco
Poucos estudos podem ser encontrados sobre a relação entre ortorexia nervosa e vegetarianismo.

Foram encontradas diferenças entre as amostras italiana e polonesa, tanto na porcentagem de indivíduos com comportamento ortoréxico, conforme sugerido pelo escore ORTO 15.

Conclui-se que um número considerável das alunas do curso de Nutrição apresenta comportamento ortoréxico e distúrbio da imagem corporal.

Salienta-se necessidade de estudos subsequentes sobre o tema, pois a relação entre transtornos interferem em seus diagnósticos, prognósticos e na elaboração de estratégias terapêuticas. 
PENAFORTE et.al, 2018

\section{MARTINS} et.al,2017

MATERA

et.al,2015

JEREZ et.al,2015 Medir a prevalência de comportamento ortoréxico em estudantes do ensino médio de uma escola particular de Temuco, Chile.

MARTINES; SOTO, 2017

FERNÁNDEZ et.al,2019
Adaptar e validar a versão em espanhol do questionário DOS para a detecção do comportamento da nervosa ortorexia.
Os estudantes com ON apresentaram mais insatisfação corporal $(\chi 2=7,48 ; p$ $=0,005)$ e excesso de peso $(\chi 2=6,56 ; p$ $=0,010)$. Não foi observada associação entre a $\mathrm{ON}$ e o período cursado.

O comportamento foi concretizado em: ganho de autoestima ao comer alimentos saudáveis $(8,8 \%)$, sensação de culpa por desviar-se da dieta $(4,6 \%)$ entre outros.

Dentro os dois grupos avaliados as principais

diferenças diziam respeito ao peso, patologia do TA e comportamentos ortoréxicos dentro do mesmo grupo de pacientes com disfunção erétil.

Observou-se que os alunos da escola municipal

obtiveram $35,3 \%$ de comportamento ortoréxico, enquanto

sujeitos da escola privada $24,7 \%$.

A experiência subjetiva das mulheres consiste em uma

campo fenomenológico que define sua historicidade: passado.
Os resultados demonstram que os estudantes de nutrição parecem ser uma população especialmente em risco para tendência à $\mathrm{ON}$ independentemente do período cursado.

Embora a (ON) ainda não seja oficialmente reconhecida como um TA, estudos sobre esse comportamento alimentar poderão embasar o seu possível futuro reconhecimento.

Diferenças e semelhanças parecem existir entre esses distúrbios e podem se beneficiar de abordagens específicas de tratamento.

Os estudantes do colégio municipal obtiveram maior prevalência em relação aos estudantes de escolas particulares em relação a ortorexia nervosa.

Construto ortorexia e os discursos corretos sobre apetite devem ser explorados em maior profundidade pelo comunidade científica para investigar: estigmatização social de pessoas preocupadas com uma alimentação saudável

Uma análise fatorial confirmatória foi aplicada na segunda metade da amostra aleatória para confirmar a solução fatorial.

A adaptação em espanhol do DOS-ES provou ser um questionário confiável e válido para avaliar a tendência obsessiva à alimentação saudável entre estudantes universitários.

A restrição cognitiva foi um preditor de ON em uma amostra que seguiu uma dieta sem carne.

contribuir para identificar fatores de risco potenciais para padrões alimentares estritos voltados para a saúde e para obter uma melhor visão sobre ON.

A maioria dos artigos aderiu à abordagem empírico-atomística, que indica a contextualização limitada dessa condição e, portanto, contribui para a representação da ortorexia como uma questão individual

Fonte: Autores

\section{Discussão}

\subsection{Ortorexia X Fatores Psicológicos}

De acordo com Cuquejo (2017), os distúrbios alimentares (TCA) são desordens na saúde mental definidos por um comportamento patológico oposta à ingestão de alimentos e uma fixação pelo controle de peso, acompanhada de distorção da concepção da imagem corporal. Sendo de origem multifatorial, incluindo causas de origem biológica, psicológica, familiar e sociocultural. Os transtornos mais conhecidos são caracterizados por anorexia nervosa e bulimia nervosa, mas 
também existem outros, como compulsão alimentar, (ON) Ortorexia Nervosa (que é a obsessão por alimentos saudáveis) e vigorexia (sendo a obsessão pelo exercício físico). Podendo causar um enfraquecimento doentio significativo.

Barrada \& Roncero (2018), afirma que os sintomas da ON são egossintônicos pois os seus hábitos e ideias alimentares são destaques centrais na vida dos pacientes. Por outro lado, os profissionais em nutrição tem uma direção de identidade, reconhecendo os transtornos alimentares como padrões comportamentais em sua ação. Sendo semelhante a definição de ON porém, tem condições suficientes para ser considerada um transtorno independente ou deve ser categorizada como um subtipo de um transtorno mental existente, como transtorno alimentar, transtorno obsessivocompulsivo, hipocondria ou mesmo um transtorno psicótico.

Porém, Martinés \& Soto (2017), destacam que embora os fatores epidemiológicos da ortorexia sejam bastante reduzidos, no estudo do autor encontrou-se por alto a magnitude do problema. Assim como, encontram-se estudos que indicam para a predominância na população em geral, estima-se em 6,9\%, e em grupos de alto risco estão (profissionais e área da saúde) entre $35 \%$ e $57,8 \%$. De outro ponto de vista, na Espanha, alguns especialistas confirmam que, entre seus pacientes o 0,5-1\%têm ortorexia.

Fernandéz (2019) concorda que, mesmo que a comunidade científica não tenha aceitado os critérios diagnósticos definitivos, alguns estudos tentaram explanar sobre o tema. Não obtendo, números significativos alcançados, até o momento, em relação a uma definição incondicional da ortorexia nervosa. Apesar disso, até o momento a ON não está incluído com um diagnóstico específico no manual primário de saúde mental, o Manual Estatístico de Transtornos Mentais (DSM-V), nem na décima edição da Classificação Internacional de Doenças (CID-10).

Haman (2015) concorda que atualmente, a ortorexia não foi admitido como uma patologia pela quinta edição do Diagnóstico e Estatística Manual de Transtornos Mentais, alguns estudiosos da mesma forma discutem se a ortorexia necessita ser considerado um distúrbio, um comportamento, ou uma prática alimentar extrema.

\subsection{Ortorexia $x$ Fatores de conhecimento}

Olejniczak et.al, (2017) retrata em seu estudo que a característica de ortorexia assemelha ter maior porcentagem entre veganos, frutarianos ou foodists crus. Como também, seu estudo assegura que entre as pessoas que comiam mais de 3 refeições por dia com mais frequência indicam que passavam mais de 3 horas por dia planejando sua dieta em comparação com as pessoas que faziam apenas 1-2 refeições, enfatizando ainda mais os sintomas da ortorexia.

Ademais, relata que, de acordo com os estudos recentes, os fatores de predisposição da ortorexia são atitudes inapropriadas quando se refere à alimentação, um distúrbio obsessivo-compulsivo e valores do índice de Massa corporal (IMC) que vão além da normalidade. Alguns dos estudos também mostram um baixo nível de escolaridade. Além disso, pessoas que já sofreram algum tipo de transtorno/distúrbio alimentar que indica expor a ortorexia com mais ênfase (Olejniczak et.al, 2017).

Estudo realizado com 283 nutricionistas austríacas do sexo feminino, mostrou que a ortorexia nervosa avaliada pelo BOT (Bratman's orthorexia test) se assemelha com mais ênfase nessas profissionais, visto que 34,9\% das participantes do estudo demonstraram certo grau de comportamento ortoréxico e 12,8\% apresentaram ortorexia nervosa (Martins et.al, 2017).

Nesse estudo o comportamento ortoréxico das nutricionistas foi assim descrito como: elevação da autoestima ao comer alimentos saudáveis $(8,8 \%)$, sentimento de culpa por fugir da dieta $(4,6 \%)$, se reprimir de comer fora de casa por ter medo uma alimentação não saudável (2,5\%), privação de comer com outras pessoas (2,5\%), e transportar os próprios alimentos quando tiver que comer fora de casa $(1,1 \%)$. Usando a mesma forma e idioma de avaliação (alemão), avaliou-se a predisposição para $(\mathrm{ON})$ em estudantes de nutrição de universidades alemãs. Foram analisados alunos do primeiro ano do 
curso $(n=123)$ e dos semestres mais adiantados $(n=96)$ com um grupo-controle de estudantes de outros cursos ( $n=68$ e $\mathrm{n}=46$ ), respectivamente (Martins et.al, 2017).

Em estudo com 404 indivíduos demonstrou que 6,9\% da amostra apresentaram Ortorexia e a doença esteve mais presente no sexo masculino, deixando assim claro que os homens tem grandes chances de desenvolver a ON (Silveira et.al, 2015). Diante disso, outro estudo verificou que $75 \%$ das universitárias mostraram distúrbio da imagem corporal, quando se é perguntado durante a aplicação da escala de silhuetas, uma figura maior do que aquela correspondente ao IMC calculado por meio dos dados aferidos. Logo, Gonçalves et al realizaram estudo comparativo com estudantes do curso de Nutrição e Educação Física, para quantificar a percepção da imagem corporal, utilizando também a escala de silhuetas, e obtendo o resultado que $75,8 \%$ dos alunos de Nutrição e 78,2\% dos alunos de Educação Física apresentaram distúrbio da imagem corporal (Souza, 2014 \& Negri, 2011).

\subsection{Ortorexia x Fatores Sociais}

Estima-se a predominância de menos de $1 \%$ da população tenham ortorexia nervosa na Espanha, sendo de forma mais acentuada em mulheres do que em homens, apresentando uma proporção de dez para um, principalmente em indivíduos vegetarianos, macrobiótica, e que tenham preferências por comidas cruas e frutas. Logo, também acredita-se que existem indícios graduais de que os fatores socioculturais e biológicos interagem entre si, contribuindo para a presença de distúrbios alimentares (Alén, 2006).

Comparou alunos de uma escola particular e outra municipal em relação aos comportamentos ortoréxicos que possivelmente poderia prevalecer, obtendo o resultado que na escola privada de $24,7 \%$ dos alunos demonstraram comportamentos ortoréxicos e quando comparados com a escola municipal 35,3\% dos alunos apresentaram comportamento ortoréxico. Quando enfatizado com a faixa etária observou-se que nos alunos de 16 anos o percentual foi de 32,9\% dos casos, aos 17 anos 27,5\% e aos 18 anos 34,8\% (Jerez, et.al , 2015).

\subsection{Ortorexia $\mathrm{x}$ fatores dietéticos}

Matera et,al, (2019) em seu estudo mostra que indivíduos que seguem uma dieta vegetariana ou vegana tem mais probabilidade a desenvolver comportamento alimentar ortoréxico do que pessoas que fazem uma dieta com proteína animal. Porém, outros estudos defendem que indivíduos que seguem uma dieta vegana obtêm uma saúde menos patológica em relação ao padrão alimentar.

Barthels; Meyer \& Pietrowski (2018), em sua pesquisa enfatizou que Veganos e vegetarianos não se diferenciam no comportamento alimentar ortoréxico, porém ambos os grupos expõe maior característica no comportamento alimentar ortoréxico do que os indivíduos que consomem carne vermelha. Logo, os autores relatam que não há diferenças em relação a esse tipo de alimentação. Pois, os indivíduos que fazem dieta com a abordagem na mudança dietética apresentam característica mais alta tanto em alimentação ortoréxica quanto com restrição alimentar, do que indivíduos sem mudança dietética e indivíduos que não fazem dieta.

Com base nos resultados obtidos de sua pesquisa ressalta que a obsessão por alimentos saudáveis é mais representativo para vegetarianos do que para não vegetarianos. Com isso, retrata que o perigo em desenvolver ortorexia nervosa e tem como efeito diminuir com o passar do tempo e a duração da dieta. Ademais, descreve em sua pesquisa que o maior número de fanáticos por alimentos saudáveis foi encontrado no grupo dos lacto-vegetarianos, um número menor entre os ovo-vegetarianos e lacto-ovo-vegetarianos, e o menor número foi observado no grupo dos veganos (Dittfeld et.al, 2017). 


\section{Considerações Finais}

A frequência de indivíduos ortoréxicos é bastante heterogênea, sendo relatados na literatura desde profissionais da área da saúde, nutricionistas, veganos, indivíduos envolvidos em causa de proteção animal, praticantes de exercícios físicos e homens. Vale salientar que são necessários mais estudos para evidenciar as populações mais vulneráveis ao ON, tal como os fatores envolvidos na gênese dessa anormalidade comportamental.

\section{Referências}

Alén, E. M Perspectiva antropológica de un caso de ortorexia nerviosa. Cultura de Los Cuidados Revista de Enfermería y Humanidades, [S.L.], n. 20, p. 109116, 2006. Universidad de Alicante Servicio de Publicaciones. http://dx.doi.org/10.14198/cuid.2006.20.15.

Andreas, S., Schedler, K., Schulz, H., \& Nutzinger, D. O. (2018). Evaluation of a German version of a brief diagnosis questionnaire of symptoms of orthorexia nervosa in patients with mental disorders (Ortho-10). Eating and weight disorders: EWD, 23(1), 75-85. https://doi.org/10.1007/s40519-017-0473-y

Aranceta Bartrina \& Javier. (2007). Ortorexia o la obsesión por la dieta saludable. Archivos Latinoamericanos de Nutrición,57(4), 313-315. http://ve.scielo.org/scielo.php?script=sci_arttext\&pid=S0004-06222007000400002\&lng=es\&tlng=es.

Barrada, Juan Ramón e Roncero María. (2018). Estrutura bidimensional da ortorexia: desenvolvimento e validação inicial de um novo instrumento. Annals of Psychology, 34 (2), 282-290. https://dx.doi.org/10.6018/analesps.34.2.299671

Barthels, F., Meyer, F. \& Pietrowsky, R. Orthorexic e comportamento alimentar restrito em veganos, vegetarianos e indivíduos em dieta. Eat Weight Disord 23, 159-166 (2018). https://doi.org/10.1007/s40519-018-0479-0

Bartrina, Javier Aranceta. Ortorexia o la obsesión por la dieta saludable. 2007. 4 f. Tese (Doutorado) - Curso de Nutrição, Departamento de Medicina Preventiva y Salud Pública. Universidad de Navarra. España, Organo Oficial de La Sociedad Latinoamericana de Nutrición, España, 2007.

Brytek-Matera, A., Czepczor-Bernat, K. \& Jurzak, H. et al. Padrões alimentares estritos voltados para a saúde (comportamentos alimentares ortoréxicos) e sua conexão com uma dieta vegetariana e vegana. Eat Weight Disord 24, 441-452 (2019). https://doi.org/10.1007/s40519-018-0563-5

Cavero, Vanessa, Lodwig, Cynthia, Lulli, Gabriela e Tejada \& Romina. (2015). Ortorexia: um novo desafio para os profissionais de saúde. Latin American Nutrition Archives, 65 (3), 1999. http://ve.scielo.org/scielo.php?script=sci_arttext\&pid=S0004-06222015000300010\&lng=es\&tlng=es.

Cuquejo, Lidia María Ortiz; Aguiar, Carlos; Dominguéz, Gloria Celeste Samudio \& Hermosilla, Avelina Troche. Eating Disorders in adolescents: a booming disease?. Pediatría (Asunción), [S.L.], v. 44, n. 1, p. 37-42, 29 abr. 2017. Sociedad Paraguaya de Pediatria. http://dx.doi.org/10.18004/ped.2017.abril.37-42.

Dell'Osso, L., Abelli, M., Carpita, B., Pini, S., Castellini, G., Carmassi, C., \& Ricca, V. (2016). Evolução histórica do conceito de anorexia nervosa e relações com a ortorexia nervosa, autismo e espectro obsessivo-compulsivo. Doença neuropsiquiátrica e tratamento, 12 , 16511660. https://doi.org/10.2147/NDT.S108912

Dittfeld A, Gwizdek K, Jagielski P, Brzęk J. \& Ziora K. A Study on the relationship between orthorexia and vegetarianism using the BOT (Bratman Test for Orthorexia). Psychiatr Pol. 2017 Dec 30;51(6):1133-1144. English, Polish. doi: 10.12740/PP/75739. Epub 2017 Dec 30. PMID: 29432508.

Fernández María Laura; Onieva-Zafra, María Dolores; Fernández-Muñoz, Juan José \& Fernández-Martínez, Elia. Adaptation and validation of the Spanish version of the DOS questionnaire for the detection of orthorexic nervosa behavior. Plos One, [S.L.], v. 14, n. 5, p. 1-11, 7 maio 2019. Public Library of Science (PLoS). http://dx.doi.org/10.1371/journal.pone.0216583.

Gonçalves, Tatiane Dutra et al. Comportamento anoréxico e percepção corporal em universitários. 2008 . 5 f. Curso de Nutrição, Departamento de Psiquiatria da Universidade de Taubaté (Unitau)., Universidade de Taubaté (Unitau)., Taubaté, 2008.

Haman Linn; Barker-Ruchti, Natalie; Patriksson, Göran \& Lindgren, Eva-Carin. Orthorexia nervosa: an integrative literature review of a lifestyle syndrome. International Journal Of Qualitative Studies On Health And Well-Being, [S.L.], v. 10, n. 1, p. 1-16, jan. 2015. Informa UK Limited. http://dx.doi.org/10.3402/qhw.v10.26799.

Jerez et al. Prevalencia de conducta ortorexica en estudiantes de educación media de Temuco. 2015.4 f. Universidad Autónoma de Chile, Sede Temuco. Temuco, Chile, 2015.

Martins, Márcia Cristina Teixeira, Alvarenga, Marle dos Santos, Vargas, Sílvia Viviane Alves, Sato, Karen Sayuri Cabral de Jesus, \& Scagliusi, Fernanda Baeza. (2011). Ortorexia nervosa: reflexões sobre um novo conceito. Revista de Nutrição, 24(2), 345-357. https://doi.org/10.1590/S1415-52732011000200015

Mateo-Martínez, Ginés \& Sellán-Soto \& Maria Carmen. (2017). En torno del apetito correcto: exploración del constructo ortorexia from los núcleos esenciales del discurso experiencial. Escola Anna Nery, 21 (3), e20160350. Epub 08 de junho de 2017. https://doi.org/10.1590/2177-9465-ean-2016-0350

Negri, Sônia Teresinha de. Influências na escolha por curso de nutrição em calouros de Porto Alegre (RS). 2011.21 f. Curso de Nutrição, Ufpel, Pelotas, 2011.

Olejniczak, D., Bugajec, D., Panczyk, M., Brytek-Matera, A., Religioni, U., Czerw, A., Grąbczewska, A., Juszczyk, G., Jabłkowska-Górecka, K., \& Staniszewska, A. (2017). Analysis concerning nutritional behaviors in the context of the risk of orthorexia. Neuropsychiatric disease and treatment, 13, 543550. https://doi.org/10.2147/NDT.S129660

Penaforte, Fernanda R. O., Barroso, Sabrina M., Araújo, Maria Eduarda, \& Japur, Camila C. (2018). Ortorexia nervosa em estudantes de nutrição: associações com o estado nutricional, satisfação corporal e período cursado. Jornal Brasileiro de Psiquiatria, 67(1), 18-24. https://doi.org/10.1590/0047-2085000000179 
Research, Society and Development, v. 11, n. 1, e127111220529, 2022

(CC BY 4.0) | ISSN 2525-3409 | DOI: http://dx.doi.org/10.33448/rsd-v11i1.20529

Silveira Jr, Luiz Alberto da Silva, Cyrino, Luiz Arthur Rangel, Zeferino, Mayara Decker, Rosa, Priscilla Cristina da, \& Borges, Sara. (2015). Ortorexia nervosa e transtorno obsessivo-compulsivo: qual a relação?. Psicologia Hospitalar, 13(2), 47-63. Recuperado em 20 de janeiro de 2021, de http://pepsic.bvsalud.org/scielo.php?script=sci_arttext\&pid=S1677-74092015000200004\&lng=pt\&tlng=pt.

Souza, Quetsia Jackeline Octacilio Vitorino de, \& Rodrigues, Alexandra Magna. (2014). Comportamento de risco para ortorexia nervosa em estudantes de nutrição. Jornal Brasileiro de Psiquiatria, 63(3), 200-204. Epub September 00, 2014.https://doi.org/10.1590/0047-2085000000026 